\title{
Resistance fluctuations in hydrogenated amorphous silicon: Thermal equilibrium
}

\author{
Paul A. W. E. Verleg and Jaap I. Dijkhuis \\ Department of Condensed Matter, Faculty of Physics and Astronomy, Debye Institute, University of Utrecht, \\ P.O. Box 80000, 3508 TA Utrecht, The Netherlands
}

(Received 23 March 1998)

\begin{abstract}
Resistance noise measurements are presented of intrinsic hydrogenated amorphous silicon films as a function of voltage and temperature in the dark. The noise displays Gaussian statistics pointing to a large number of independent noise sources. It is demonstrated that the spectral dependence and temperature dependence are the result of a large number of independent thermally activated fluctuators. A distribution of activation energies for the characteristic rates is obtained from the measurements that peak at $0.85 \mathrm{eV}$ with a full width at half maximum of $\sim 0.2 \mathrm{eV}$. The attempt rate amounts to $7 \times 10^{12} \mathrm{~s}^{-1}$. We discuss general mechanisms for mobility fluctuations and number fluctuations due to generation and recombination of free carriers. A model for generation-recombination noise in a semiconductor with localized electronic states distributed throughout the mobility gap appears consistent with both the variance of the fluctuations and the temperature dependence of the rate constants. We identify the activation energy and attempt rate as the barrier limiting thermal emission of holes from defect states to the valence band. The width of the distribution of activation energies points to inhomogeneous broadening due to band bending. Light-induced metastable changes in the material are observed to affect the variance quite significantly. The present measurements and analysis of the noise under equilibrium conditions pave the way to successfully examine nonthermal equilibrium fluctuations in the photoconductivity and under injection conditions, which is the subject of the accompanying paper in this volume. [S0163-1829(98)07031-3]
\end{abstract}

\section{INTRODUCTION}

Hydrogenated amorphous silicon $(a-\mathrm{Si}: \mathrm{H})$ has drawn the attention of many researchers over the years, not only driven by the interest $a$-Si:H has in technological applications but also as a model system for understanding the physics of amorphous semiconductors. Indeed, $a-\mathrm{Si}: \mathrm{H}$ is of fundamental interest as it displays a wide range of physical phenomena that are not encountered in crystalline semiconductors. In particular the dynamics of charge carriers and the effect of light-induced degradation (the Staebler-Wronski effect ${ }^{1}$ ) have received a lot of attention. ${ }^{2}$ Despite this effort, however, the detailed mechanisms of defect creation, hydrogen motion, and electron-hole recombination remain unclear. A common property of these phenomena is that they are expected to give rise to resistance fluctuations. If strong enough, i.e., exceeding thermal noise, ${ }^{3}$ these fluctuations can be measured and contain new information on the dynamics of the underlying electronic or chemical processes.

In the last decade, the first reports were published on noise in $a-\mathrm{Si}: \mathrm{H}$. These studies can roughly be divided into three groups on the basis of device geometry and the material studied. Parman et al. ${ }^{4-7}$ extensively studied noise in doped $n$-type $a$-Si:H with coplanar contact geometry. The authors found strong evidence that in these devices part of the current is carried by inhomogeneous current filaments, whose resistance is sensitive to local atomic rearrangements. Fan and Kakalios et al. $^{8}$ proposed a model in which hydrogen motion results in modulation of the conductivity of these microchannels. In amorphous-silicon Schottky barriers, one invariably encounters random telegraph noise. ${ }^{9-13}$ Switching amplitudes as high as $20 \%$ of the total resistance have been observed. The random telegraph noise seems to be well explained by a modulation of the conductance of a transport path through the tunnel barrier due to fluctuations of the barrier height induced by trapping and detrapping of charge carriers at nearby traps. There have been a few studies only, of noise in intrinsic amorphous-silicon devices with a sandwich geometry. Baciocchi, D'Amico, and van Vliet ${ }^{14}$ studied $\mathrm{Cr}-i$ - $\mathrm{Cr}$ devices with an intrinsic layer between 200- and 500 -nm thick. The spectra are close to $1 / f$. A tentative explanation has been given in which the dangling bond concentration was allowed to fluctuate with a distribution of activation energies. However, no evidence of thermally activated kinetics was given. Bathaei and Anderson ${ }^{15}$ carried out noise measurements on $\mathrm{Cr}-n^{+}-i-n^{+}-\mathrm{Cr}$ devices with a $2-\mu \mathrm{m}$-thick intrinsic $a$-Si:H layer. They concluded that the low-frequency noise was produced by electron emission from the traps to the conduction band. Clearly, the origin for noise in intrinsic $a-\mathrm{Si}: \mathrm{H}$ is still an open question.

We present a comprehensive study of noise in intrinsic $a-\mathrm{Si}: \mathrm{H}$ and aim at elucidating the origin of the resistance fluctuations. In this paper we exclusively consider thermal equilibrium resistance fluctuations, i.e., we examine the noise in the dark and in a regime where the current-voltage characteristic displays Ohmic behavior. In the next paper we extend our measurements to nonthermal equilibrium situations and study noise in the photoconductivity and under conditions of carrier injection.

This paper is organized as follows. In Sec. II the devices and the experimental setup are described. In the Appendix we explain how bulk contributions to the noise could be separated from spurious noise sources located in the contact regions. The main results are described in Sec. III. We have measured noise spectra in the dark as a function of voltage and temperature. We have checked that the noise is Gaussian, indicating the presence of a large number of independent fluctuators. The fluctuators all display thermally activated ki- 
netics. Finally, we present results that demonstrate the influence of metastable defect creation on the noise intensity. In Sec. IV we analyze our data phenomenologically using the Dutta-Dimon-Horn approach ${ }^{22}$ and derive a distribution of activation energies for the rates of the fluctuations consistent with all our noise data. In an attempt to identify the physical origin of the noise we discuss in Sec. V the important general mechanisms that produce noise in $a-\mathrm{Si}: \mathrm{H}$ and in relation to existing literature on noise spectroscopy of $a-\mathrm{Si}: \mathrm{H}$. A model for generation-recombination (GR) noise involving a continuous distribution of localized electronic states appears to be consistent with the majority of our observations. The analysis yields the attempt rate for thermal emission of a hole from a defect state to the valence band. Decisive evidence for GR noise in $a$-Si:H will be presented in the next paper where the noise measured under nonthermal equilibrium conditions is discussed.

\section{EXPERIMENT}

\section{A. Devices}

We use Cr- $n^{+}-i-n^{+}-\mathrm{Cr}$ structures with a $1-\mu \mathrm{m}$-thick intrinsic $a$-Si:H layer sandwiched between two 50-nm-thick, highly doped $n$-type layers to provide optimal contacts to the chromium metal films. The circular top contact has a diameter of $3 \mathrm{~mm}$ and is only $15-\mathrm{nm}$ thick to allow for illumination of the intrinsic layer by visible light. The sandwich geometry was found to be the best compromise for a detailed study of noise in intrinsic $a$-Si:H over a wide range of temperatures. The device-quality $a-\mathrm{Si}: \mathrm{H}$ films were deposited on Corning 7059 glass using plasma enhanced chemical vapor deposition (PECVD) at a substrate temperature of $470 \mathrm{~K}$. Special attention was paid to device preparation to obtain the best possible contacts: The metal films were deposited in vacuum better than $5 \times 10^{-7}$ mbar in a clean-room environment. In order to minimize oxide growth, all processing steps were carried out without interruption. Despite all these efforts, one may not bluntly assume that our devices have "ideal" electron injecting contacts. As chromium and intrinsic $a$-Si:H have different work functions, strong band bending occurs when the metal and semiconductor are connected, resulting in charge accumulation predominantly at the semiconductor side of the interface. Such contact effects are, of course, reduced by employing the highly doped, low-Ohmic buffer layer between the chromium contact and the intrinsic $a$-Si:H film. Even under these conditions some band bending occurs as illustrated in Fig. 1. In this figure we plot the results of a simulation of the position-dependent conduction band and valence band for an $n^{+}-i-n^{+}$device using device and semiconductor parameters typical for our samples. Although the absolute numbers should be taken only as approximate, this graph shows that the Fermi level $E_{F}$ at the $n^{+}-i$ and $i-n^{+}$interfaces lies $\sim 0.25 \mathrm{eV}$ below the electron mobility edge $E_{c}$, whereas in the bulk of the device $E_{c}$ $-E_{F} \sim 0.75 \mathrm{eV}$. We still expect the device to display Ohmic behavior at low fields though, since the relatively highresistive intrinsic part of the device will limit the conductance. Nevertheless, we also see that trapped space charge does influence the device conductance, since in pure intrinsic material one generally finds $E_{c}-E_{F} \sim 0.85 \mathrm{eV}$ instead of the

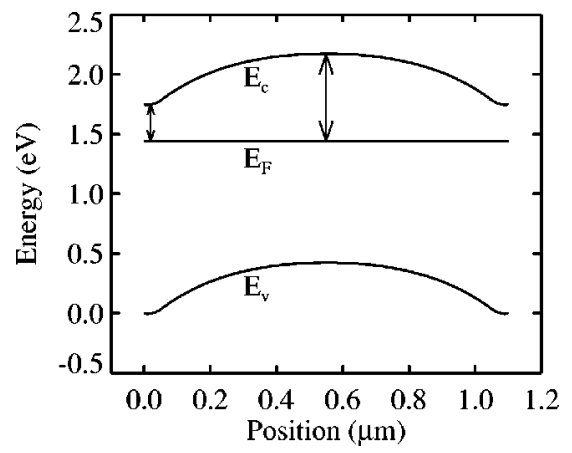

FIG. 1. Simulation of the position-dependent energy-band diagram for an $a-\mathrm{Si}: \mathrm{H} n^{+}-i-n^{+}$sandwich structure.

$0.75 \mathrm{eV}$ from Fig. 1. It is important to account for this fact in the interpretation of the noise properties of $a-\mathrm{Si}: \mathrm{H}$ sandwich structures.

Six devices from two different batches have been studied in detail. The results within one batch appeared to be reproducible over many months within experimental error. Between batches, the noise differed by at most a factor of two. In all cases, the qualitative dependence of the noise on various parameters was identical. Further, the results did not depend on the current direction. We conclude that the contribution of the noise from the contacts is negligible in the range of temperatures studied, and that the results pertain to genuine intrinsic $a$-Si:H resistance noise. Finally, we note that the Appendix deals with the high-temperature and highelectric-field noise phenomena that possibly originate from the contact regions or the doped $a-\mathrm{Si}: \mathrm{H}$ layers. The intensity of this type of noise is found to vary considerably from sample to sample and will not be touched on any further, since it is not of intrinsic nature.

\section{B. The setup}

The device is glued on a copper block using a thermally conductive paste and located in a temperature controlled vacuum vessel with optical and electrical access. The temperature can be varied from 77 to $575 \mathrm{~K}$ by means of three exchangable vacuum chambers. Around room temperature we use a Peltier device to control the device temperature. The low- and high-temperature regimes are covered using resistive heaters. Below $250 \mathrm{~K}$ the sample block is mounted on a cold finger in a liquid nitrogen cryostat.

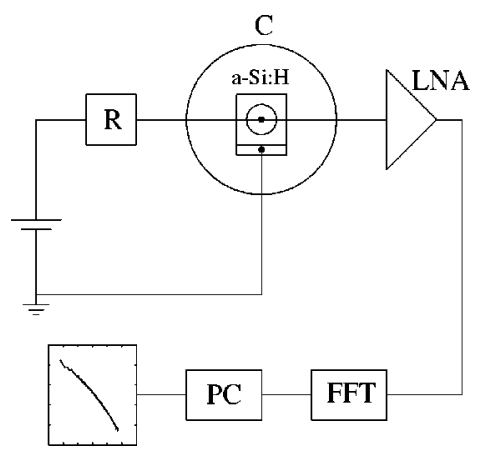

FIG. 2. Schematic representation of the experimental setup. $R$ denotes series resistance, $C$ vacuum chamber, LNA low noise amplifier, FFT digital spectrum analyzer, and PC personal computer. 


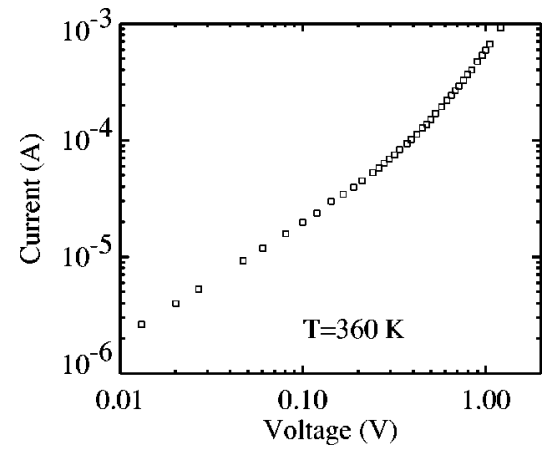

FIG. 3. Typical current-voltage characteristic of the amorphous silicon $\mathrm{Cr}-n^{+}-i-n^{+}-\mathrm{Cr}$ samples.

During a typical noise measurement a constant current is driven by fully charged dry batteries through the sample in series with a resistance that is always at least 20 times larger than that of the sample (see Fig. 2). The constant current serves to convert spontaneous resistance fluctuations into voltage fluctuations. The fluctuating voltage over the sample is amplified using an NF Electronics low-noise voltage amplifier (LI 75A). The autocorrelation spectrum of the amplifier output signal was determined between $0.25 \mathrm{~Hz}$ and 100 $\mathrm{kHz}$ using a computer-controlled digital spectrum analyzer (Advantest R9211A). Special care was taken in shielding and grounding of the setup to eliminate rf pickup. Prior to all measurements the sample was annealed at $440 \mathrm{~K}$ and slowly cooled to room temperature. The measurements at elevated temperatures were always carried out from room temperature upwards. In the same run, we also performed nonthermal equilibrium noise measurements under illumination, which are discussed in the following paper. The illumination intensities were always very low and never exceeded $10 \mu \mathrm{W} \mathrm{cm}{ }^{-2}$ to minimize degradation effects. All noise measurements include a background noise measurement with no current running through the sample to determine the socalled excess noise. The response of the setup is frequency independent for all data that are presented here.

\section{RESULTS}

\section{A. Current-voltage characteristics}

In Fig. 3 we plot a typical current-voltage characteristic. It is Ohmic at low voltages but becomes superlinear for higher voltages pointing to space-charge-limited currents. In the Ohmic regime, the conductivity is thermally activated with an activation energy of $0.73 \mathrm{eV}$. This is somewhat smaller than usually observed in thick intrinsic films but consistent with the discussion around Fig. 1, where we show that the deviation can be traced back to electron injection by the doped $a$-Si:H contact layer.

\section{B. Power spectra}

All experimental results presented in this paper were obtained in the dark. In Fig. 4 we plot the relative voltage noise power $S_{V} / V^{2}$, measured at a frequency of $10 \mathrm{~s}^{-1}$ versus $V$. $S_{V} / V^{2}$ is constant at low voltages where the current-voltage characteristic is Ohmic, demonstrating that we are dealing with resistance fluctuations. In the presence of a bias current,

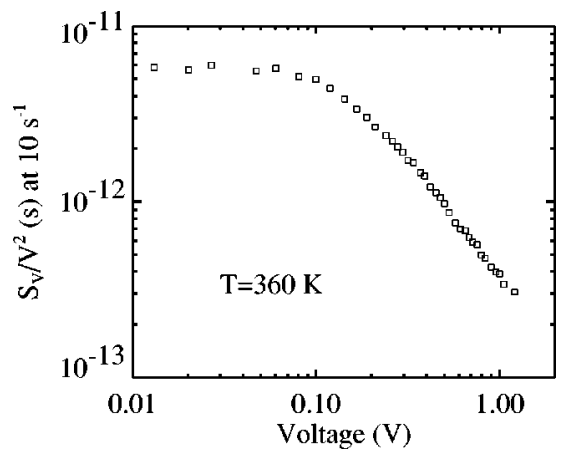

FIG. 4. Relative noise power at $10 \mathrm{~s}^{-1}$ vs voltage, in the Ohmic regime independent of current.

fluctuations in the resistance, $\delta R$, are readily converted into voltage fluctuations, $\delta V$, via Ohm's law: $\delta V=I \delta R$. Then, for the power spectrum of the voltage fluctuations we obtain

$$
S_{V}(f)=I_{0}^{2} S_{R}(f)=V_{0}^{2} \frac{S_{R}(f)}{R^{2}} .
$$

This explains that $S_{V} / V^{2}$ is constant and indicates that the current flow does not induce the resistance fluctuations but exclusively acts as a probe. At high voltages, however, $S_{V} / V^{2}$ decreases. We will address this suppression effect in the next paper where nonthermal equilibrium situations are discussed as they exist under current injection and illumination. All noise measurements were taken in the Ohmic regime, i.e., at $\leqslant 50$ - $\mathrm{mV}$ sample bias.

A typical example of a noise power spectrum is presented in Fig. 5. This spectrum was taken at $403 \mathrm{~K}$ and is the result of averaging 200 individual power spectra. Note that again the intensity of the relative voltage fluctuations $S_{V} / V^{2}$ is plotted. The spectrum has a $1 / f^{\nu}$ shape where $\nu$ depends on frequency. For comparison also a pure $1 / f$ spectrum and a Lorentzian spectrum are shown.

The pure $1 / f$ spectrum can be the result of thermally activated processes over barriers with a featureless distribution of heights. The other extreme, a single activation energy $E_{a}$ with attempt frequency $\omega_{0}$, however, produces a Lorentzian contribution, i.e., $\propto 1 / f^{2}$ above the characteristic frequency

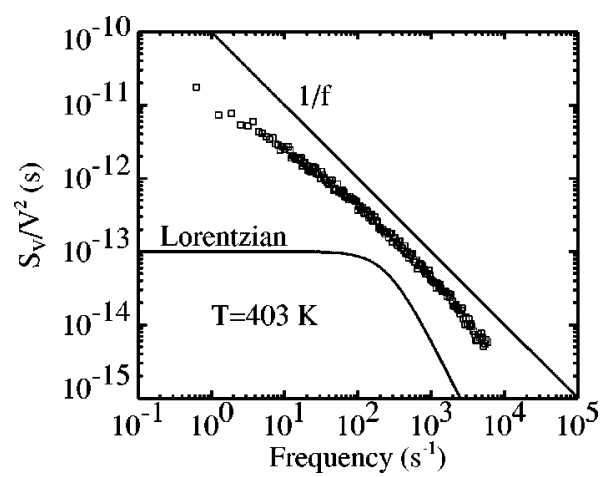

FIG. 5. Intensity of the relative voltage fluctuations vs frequency. This spectrum is the result of averaging 200 individual power spectra. Correction for thermal and background noise was made. The spectral response of the setup is frequency independent for the data shown. For comparison, a Lorentzian and a pure $1 / f$ spectrum are shown (full lines). 


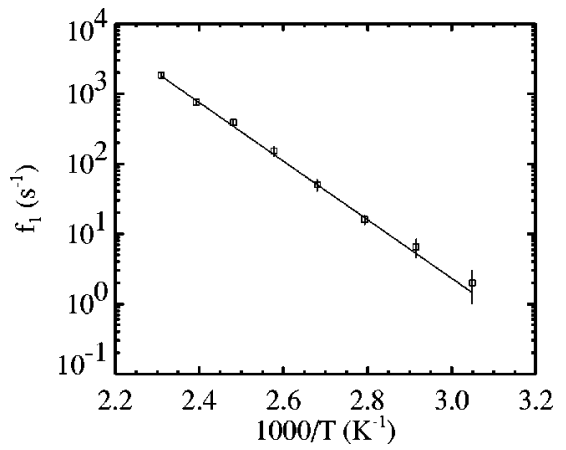

FIG. 6. Frequency at which the slope of the spectrum equals 1 vs temperature. Activation energy is $0.83 \mathrm{eV}$ and attempt rate is $7 \times 10^{12} \mathrm{~s}^{-1}$.

$f_{1}=f_{0} \exp \left[-E_{a} /\left(k_{B} T\right)\right]$. Our measurements suggest an intermediate case in our samples. For a broad, but not arbitrarily broad distribution of activation energies, one expects the spectral slope $\nu$, defined locally as $\nu=-d \ln S_{V} / d \ln f$, to depend on frequency. The characteristic frequency where $\nu$ equals 1 corresponds to the energy at which this distribution is flat, i.e., its maximum. We have plotted the characteristic frequency $f_{1}$ vs $T$ between 290 and $420 \mathrm{~K}$ in Fig. 6 . The data are fitted to

$$
f_{1}=f_{0} e^{-E_{p} /\left(k_{B} T\right)} .
$$

The errors indicate uncertainties in the fitting procedure. We obtain for the attempt rate $(7 \pm 4) \times 10^{12} \mathrm{~s}^{-1}$ and the energy at which the distribution of activation energy peaks $E_{p}$ $=0.83 \pm 0.02 \mathrm{eV}$. The errors indicate uncertainties that follow from the fitting procedure.

\section{Noise statistics}

The average spectral densities measured so far (see Fig. 5) are the Fourier transforms of the two-point voltage-voltage correlation function. ${ }^{3}$ For $1 / f$ noise in most systems no additional information is obtained by studying higher-order correlation functions, i.e., the noise in the noise. If not only the voltage but also the noise intensity varies with time, information is lost if one only studies the time-averaged spectrum. In that case the noise has non-Gaussian properties. ${ }^{16,17}$ An example of non-Gaussian noise is random telegraph noise (RTN), when the resistance switches between two discrete levels due to a single fluctuator. For RTN, the noise intensity at different frequencies is strongly correlated and the power spectrum has a Lorentzian shape. As soon as many identical fluctuators are present, acting independently, the noise intensity at different frequencies is uncorrelated, while the spectral shape remains the same.

Parman et al. ${ }^{4-7}$ and Fan and Kakalios ${ }^{8}$ studied noise in doped $n$-type $a$-Si:H devices with coplanar geometry. The noise statistics was found to exhibit very clear non-Gaussian effects indicative of strongly interacting noise sources. ${ }^{16,17}$ We shall now demonstrate that our sandwich devices show Gaussian statistics. We measured 500 time traces consisting of 256 data points. Each of the time traces is fast Fourier transformed and stored by a computer. The discrete Fourier points of every spectrum are summed into seven octave bins. The first octave contains only one point, the seventh contains
TABLE I. Normalized covariance matrix for the voltage noise of a $100-\mathrm{k} \Omega$ metal film resistor at room temperature measured from 500 individual power spectra. Frequency range covered is $5-640 \mathrm{~s}^{-1}$.

\begin{tabular}{lccccccr}
\hline \hline Octave & 1 & 2 & 3 & 4 & 5 & 6 & 7 \\
\hline 1 & 1 & & & & & & \\
2 & 0.04 & 1 & & & & & \\
3 & 0.02 & 0.10 & 1 & & & & \\
4 & -0.01 & 0.02 & 0.08 & 1 & & & \\
5 & 0.07 & 0.05 & -0.02 & 0.01 & 1 & & \\
6 & 0.01 & -0.01 & 0.01 & 0.04 & -0.03 & 1 & \\
7 & 0.01 & -0.04 & 0.05 & -0.01 & -0.02 & -0.02 & 1 \\
\hline \hline
\end{tabular}

64 points. The correlation coefficients $C_{i j}$ between octaves $i$ and $j$ can be calculated using the expression

$$
C_{i j}=\frac{1}{Q} \sum_{q=1}^{Q} \frac{\left(P_{i}-\overline{P_{i}}\right)\left(P_{j}-\overline{P_{j}}\right)}{\sigma_{i} \sigma_{j}},
$$

where $Q$ is the number of spectra, $P_{i}$ is the noise power of octave $i, \overline{P_{i}}$ is the average noise power in that octave by averaging $Q$ power spectra, $\sigma_{i}$ is the standard deviation, and the summation is over all spectra. The values of $C_{i j}$ range from +1 for completely correlated octaves to -1 for anticorrelated octaves. Ideally, for Gaussian noise, $C_{i j}=0$ for $i$ $\neq j$. We note that due to the finite number of samples we have taken the cross correlations between the fluctuations in the noise power in the different octaves amount to $0 \pm 0.05$.

In Table I the covariance matrix is shown for thermal noise of a $100-\mathrm{k} \Omega$ metal film resistor at room temperature, a typical Gaussian noise source. The autocorrelation coefficients equal 1, by definition. The off-diagonal elements are close to zero with both positive and negative values. These results show that the analysis works properly.

In Table II the covariance matrix of the correlation coefficients is shown for noise in a biased $a-\mathrm{Si}: \mathrm{H}$ sample at 343 $\mathrm{K}$. Again, we observe that the values of the off-diagonal elements are close to zero with both positive and negative values. We therefore conclude that within the accuracy of the experiment the noise is Gaussian, which means that the noise is produced by a large number of independent noise sources. Consequently, all information that can be extracted from the

TABLE II. Normalized covariance matrix for the voltage noise of an $a$-Si:H sample at $343 \mathrm{~K}$ measured from 500 individual power spectra. Frequency range covered is $1-128 \mathrm{~s}^{-1}$. For pure Gaussian noise the cross correlation between the fluctuations in the noise power in the different octaves would be $0 \pm 0.05$.

\begin{tabular}{lccccccc}
\hline \hline Octave & 1 & 2 & 3 & 4 & 5 & 6 & 7 \\
\hline 1 & 1 & & & & & & \\
2 & 0.06 & 1 & & & & & \\
3 & 0.04 & 0.04 & 1 & & & & \\
4 & -0.01 & 0.05 & -0.01 & 1 & & & \\
5 & 0.03 & 0.07 & 0.05 & 0.10 & 1 & & \\
6 & -0.03 & -0.01 & 0.08 & -0.09 & -0.07 & 1 & \\
7 & -0.01 & 0.04 & -0.01 & -0.02 & -0.01 & 0.10 & 1 \\
\hline \hline
\end{tabular}




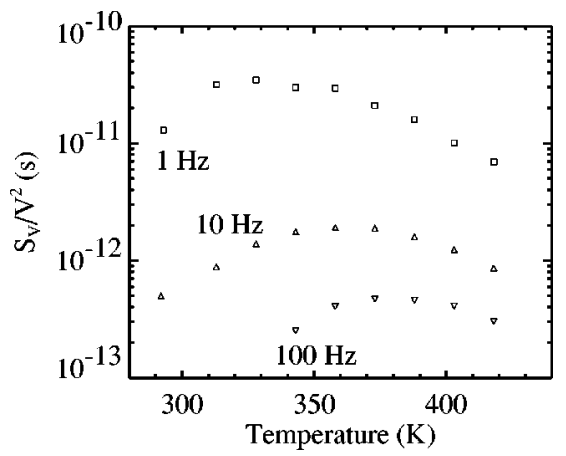

FIG. 7. Relative noise power vs temperature for three measuring frequencies. Note the shift of the broad peaks to higher temperatures for larger frequencies.

noise is contained in the two-point correlation function. Deviations from Gaussian behavior are expected only in case there is a small number of independent noise sources or a large number of highly cooperative noise sources. Our results in sandwich structures are different from those obtained by Parman $e t a l$. in coplanar devices of doped $n$-type $a$-Si:H. They found very large correlation coefficients. Further, in our case the characteristic frequency displays thermally activated behavior, which was not observed in coplanar devices. We conclude that the noise in our sandwich devices has a totally different origin. This is not to say that these studies necessarily contradict. First of all, they have studied doped $n$-type $a$-Si:H whereas we study intrinsic $a$-Si:H. Khera et $a l .{ }^{18}$ also studied noise in intrinsic $a$-Si:H but only at very high temperatures $(\geqslant 450 \mathrm{~K})$. At these temperatures we also observe non-Gaussian effects (see the Appendix). Further, different device geometries may cause different noise sources to predominate: The ratio of contact area to contact separation of the coplanar devices is six orders of magnitude smaller than of our sandwich devices. This results in much lower current densities in our devices. Johanson, Scansen, and Kasap have reported that the current density influences the noise properties of coplanar devices. ${ }^{19}$ Further, current filaments have been invoked to explain noise in coplanar devices. RTSN is then induced by changes in the local atomic structure that influence the conductivity of these microchannels. In sandwich devices there will be an orders of magnitude larger number of current filaments but with orders-of-magnitude-shorter lengths. Therefore, even if current filaments exist in our samples and the noise mechanism would be the same, the statistics of the fluctuations should in any case be Gaussian.

\section{Temperature dependence}

In Fig. 7 we present the relative noise power versus $T$ at 1,10 , and $100 \mathrm{~s}^{-1}$. All three data sets show a clear maximum, instead of rising linearly with temperature, as would be the case for pure $1 / f$ noise. The maximum shifts to higher temperatures with increasing measuring frequency in line with our earlier observation of thermally activated kinetics, as we will explain now. Let us consider a single noise source with a thermally activated rate constant $1 / \tau$ and a Lorentzian spectrum

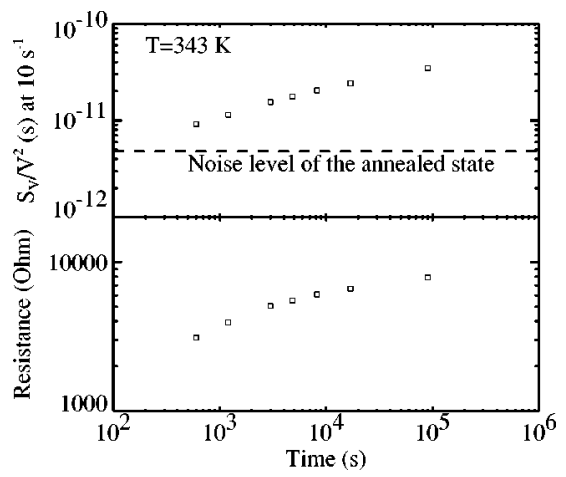

FIG. 8. Relative noise intensity and resistance vs total illumination time, i.e., degree of degradation of the sample, indicating that the noise is sensitive to the presence of (metastable) defects.

$$
S_{i}(f) \propto \frac{\tau}{1+4 \pi^{2} f^{2} \tau^{2}} .
$$

This function peaks at a temperature given by

$$
T_{p}=-\frac{E_{a}}{k_{B}} \frac{1}{\ln \left(f / f_{0}\right)} .
$$

As long as $f \ll f_{0}$ the peak temperature indeed shifts to higher temperatures with frequency.

\section{E. Degradation}

An internal parameter that can be easily controlled in $a-\mathrm{Si}: \mathrm{H}$ is the defect density. Defects are created by illuminating the sample through the semitransparent top contact. These defects can be removed again by heating the sample to $\sim 470 \mathrm{~K}$. To study the effects of degradation, the sample was first annealed to annihilate defects created during earlier light exposures and then illuminated by a heat-filtered halogen light source at an intensity of approximately $2 \mathrm{~mW} \mathrm{~cm}^{-2}$. At regular intervals, illumination was stopped and the sample left in the dark to allow for the structure and electronic system to reach a new equilibrium state. We measured the noise intensity in the dark as a function of exposure time. The result is presented in the upper panel of Fig. 8. The lower panel shows the sample resistance in the dark vs exposure time. To accelerate the degradation process, the final point at the right of the figure is obtained after exposure with an eleven times higher intensity. Correspondingly, the exposure time at this intensity has been multiplied with a factor of eleven. Of course, a longer exposure time at low intensities is generally not equivalent to a short exposure time at a higher intensity, ${ }^{20}$ but this picture only serves to show the systematic increase of $S_{V} / V^{2}$ together with the resistivity. We checked that $S_{V} / V^{2}$ reduces to its original level when the sample is annealed at $\sim 440 \mathrm{~K}$.

\section{ANALYSIS}

As we have seen, the data show all signs of thermally activated kinetics. Two central questions now arise for a proper analysis of the data: (i) What is the shape of the distribution of activation energies? (ii) Are the observed temperature dependence and frequency dependence of $S_{V} / V^{2}$ 


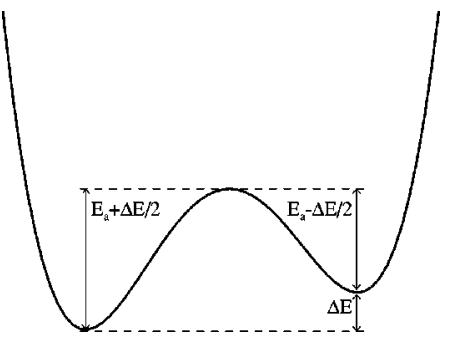

FIG. 9. Two-state system characterized by a well offset $\Delta E$ and barriers $E_{a} \pm \Delta E / 2$ for making transitions from one state to the other.

consistent with a single distribution of activation energies?

To address these questions, we introduce the DuttaDimon-Horn approach, which enables us to extract a phenomenological activation-energy distribution consistent with both the spectral and temperature dependence of the noise intensity.

\section{A. Dutta-Dimon-Horn approach}

For a random process with one characteristic time constant, the power spectrum is Lorentzian [see Eq. (4)]. An obvious way to generate a $1 / f^{\nu}$ spectrum is to superimpose a large number of Lorentzians with a suitable distribution $D(\tau)$ of characteristic times $\tau .^{21}$ For thermally activated random processes, Dutta, Dimon, and Horn ${ }^{22}$ developed a model to extract, under certain restrictions, an energy barrier distribution from a given spectrum. A pure $1 / f$ spectrum emerges when a flat distribution of activation energies is present. In general, more complicated distributions can be encountered.

Let us consider a single two-state system with an energy difference between the two states, $\Delta E$, and a barrier $E_{a}$ $\pm \Delta E / 2$ for making the transition from left to right or vice versa (see Fig. 9). In a review article by Weissman on $1 / f$ noise $^{23}$ it is shown that each two-state system contributes a Lorentzian to the spectrum of the form

$$
S(f, T)=\frac{1}{\cosh ^{2}\left[\Delta E /\left(2 k_{B} T\right)\right]} \frac{\tau}{1+4 \pi^{2} f^{2} \tau^{2}},
$$

with the effective rate given by

$$
\frac{1}{\tau}=\frac{1}{\tau_{0}}\left(e^{-\left(E_{a}+\Delta E / 2\right) /\left(k_{B} T\right)}+e^{-\left(E_{a}-\Delta E / 2\right) /\left(k_{B} T\right)}\right) .
$$

Here, $1 / \tau_{0}$ is an attempt to escape frequency, $k_{B}$ Boltzmann's constant, and $T$ the temperature. $1 / \tau$ is not exactly of the Arrhenius type but approaches it for $\Delta E \ll E_{a} . S(f, T)$ depends on temperature both through the variance in the occupation of a state, $1 / \cosh ^{2}\left[\Delta E /\left(2 k_{B} T\right)\right]$, and the activated character of $1 / \tau_{\text {eff }}$.

In a collection of two-state systems $E_{a}$ and $\Delta E$ are distributed according to $D\left(E_{a}, \Delta E\right)$. A suitable $D\left(E_{a}, \Delta E\right)$ may give the required $1 / f^{\nu}$ spectrum. In order to obtain $1 / f^{\nu}$ noise one needs a sufficiently broad distribution of activation energies $D_{1}\left(E_{a}\right)$ where

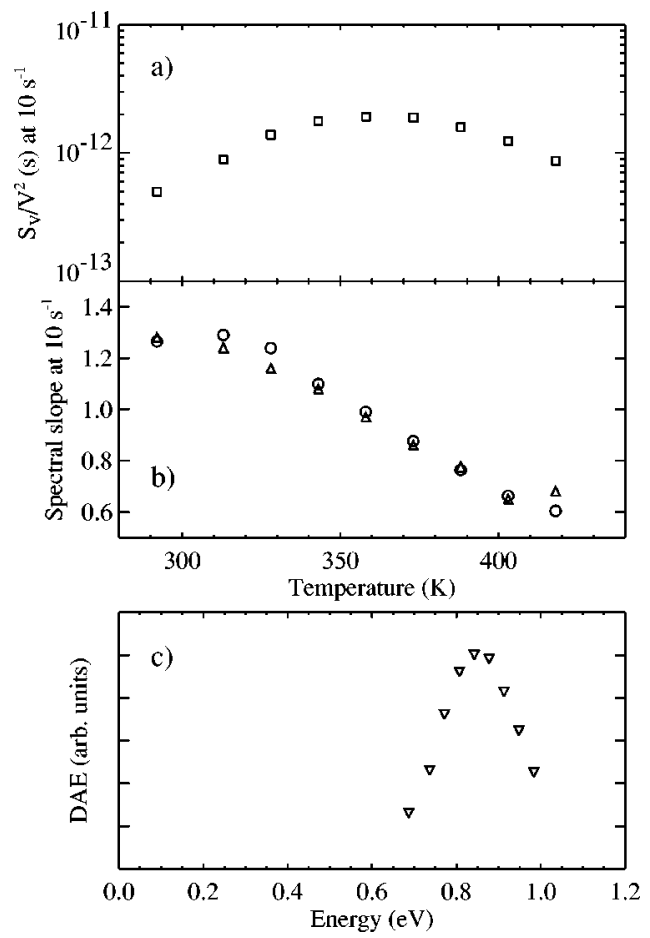

FIG. 10. (a) $S_{V} / V^{2}$ vs temperature, (b) measured spectral slope vs temperature $(\bigcirc)$ compared with $\mathrm{DDH}$ result $(\triangle)$, and (c) distribution of activation energies (DAE) derived from the data.

$$
D_{1}\left(E_{a}\right)=\int_{-\infty}^{\infty} D\left(E_{a}, \Delta E\right) \frac{1}{\cosh ^{2}\left[\Delta E /\left(2 k_{B} T\right)\right]} d \Delta E .
$$

The integral essentially selects the leading two-state fluctuators at a given temperature. Since the shape of the spectrum and the temperature dependence of the noise are produced by the same distribution of activation energies, the two are connected. Dutta, Dimon, and Horn ${ }^{22}$ derived a simple relation between the slope of the spectrum and the temperature dependence of the noise intensity. They explicitly assumed that the fluctuations have (i) Arrhenius type of thermally activated kinetics $\left(\Delta E \ll E_{a}\right)$, (ii) a single attempt rate $f_{0}$ much larger than the measuring frequency, but $E_{a}$ may vary, and (iii) a temperature-independent coupling to the resistance. Now, for a slowly varying $D_{1}\left(E_{a}\right)$ on the scale of $k_{B} T$ it can be shown that ${ }^{24}$

$$
S(f, T) \propto \frac{k_{B} T}{f} D_{1}\left(E_{a}\right),
$$

with $E_{a}=-k_{B} T \ln \left(f / f_{0}\right)$ the value of $E_{a}$ where Eq. (4) peaks. Clearly, when $D_{1}\left(E_{a}\right)$ is constant an exact $1 / f$ spectrum is obtained. However, in any other case, $S(f, T)$ is a complex function of $f$ and $T$. The relevant question to address is whether the observed dependence of the noise intensity on $f$ at a constant temperature and on $T$ at a constant frequency are consistent with each other, because then we may describe the noise sources simply by a single distribution of activation energies. This criterion can be cast in the so-called DuttaDimon-Horn (DDH) relation. It connects the slope $\nu$ of the spectrum, locally defined as $-d \ln S(f, T) / d \ln f$, to the temperature dependence of $S(f, T)$ and reads 


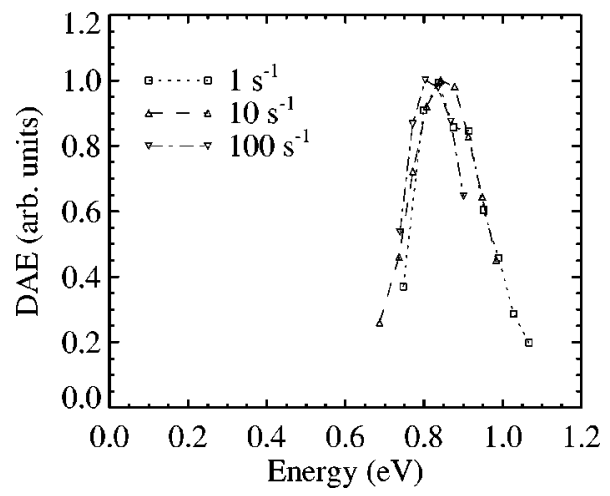

FIG. 11. Calculated distribution of activation energies (DAE) obtained from the three data sets shown in Fig. 7 and from Eq. (9). Curves are normalized to the peak height.

$$
\nu(f, T)=1-\frac{1}{\ln \left(f / f_{0}\right)}\left(\frac{d \ln S(f, T)}{d \ln T}-1\right) .
$$

Here, $S(f, T)$ represents the relative power spectral density $S_{V} / V^{2}$.

In Fig. 10(a) we plot once again $S_{V} / V^{2}$ at $10 \mathrm{~s}^{-1}$ vs temperature (cf. Fig. 7). From each spectrum (see Fig. 5) the slope $\nu$ was determined at $10 \mathrm{~s}^{-1}$. The result is shown in Fig. 10(b) (open circles). The spectral slope varies from 1.3 to 0.6 over the temperature range studied. This is at variance with results reported by Bathaei and Anderson ${ }^{15}$ where the slope becomes steeper with increasing temperature. From the measured relative noise power at $10 \mathrm{~s}^{-1}$ versus $T$ [Fig. 10(a)] and the Dutta-Horn equation [Eq. (10)] we calculated the slope as predicted by this model (Fig. 10, open triangles) using for $1 / \tau_{0}$ the value of $7 \times 10^{12} \mathrm{~s}^{-1}$ as obtained from Fig. 6. Interestingly, the spectral slope predicted by the DDH relation virtually coincides with the spectral slope measured. We therefore conclude that the frequency and temperature dependence of $S_{V} / V^{2}$ can be traced back to a single distribution of thermally activated processes. We note that this circumstance is not met in all samples: below we shall consider data sets for which the DDH relation does not hold exactly.

We are now in a position to extract $D_{1}\left(E_{a}\right)$ from our data with the help of Eq. (9). In Fig. 10(c) we show the result. $D_{1}\left(E_{a}\right)$ peaks around $0.85 \mathrm{eV}$, in agreement with the result obtained from Fig. 6, and has a width of approximately 0.2

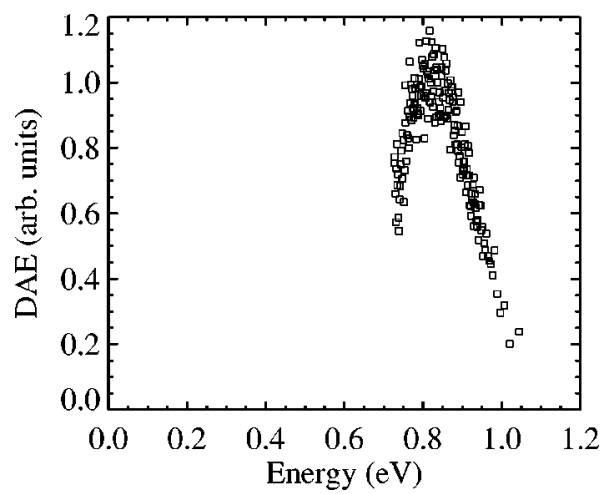

FIG. 12. Distribution of activation energies (DAE) calculated from the spectrum shown in Fig. 5.

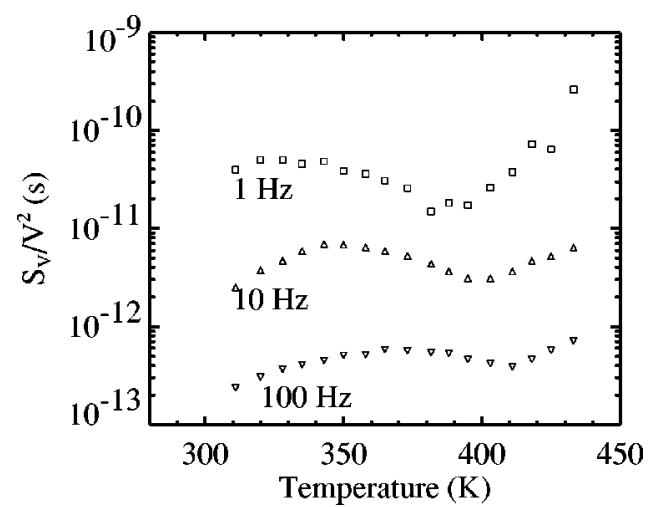

FIG. 13. Temperature dependence of the relative noise power measured at three different frequencies.

$\mathrm{eV}$. Clearly the width is much larger than $k_{B} T$ as required for the approximation [Eq. (9)]. Since the position of the peak is given by

$$
E_{p}=-k_{B} T_{p} \ln \left(f / f_{0}\right),
$$

and $E_{p}$ is a constant material parameter the model predicts that the temperature $T_{p}$ where $S_{V} / V^{2}$ peaks must depend on the measuring frequency. This, indeed, is observed in our experiments (see Fig. 7) and we can again calculate using Eq. (9) the distribution of activation energies. As Fig. 11 shows, the results obtained agree with the $10-\mathrm{s}^{-1}$ data, as expected. We have chosen quite arbitrarily to calculate $D_{1}\left(E_{a}\right)$ from the temperature dependence of the relative noise power at a fixed frequency. Of course, according to Eq. (9) the same result follows from any single spectrum. In Fig. 12 we show that this is indeed the case.

For this particular data set the DDH analysis worked out very well. As already noted, deviations are encountered from the DDH prediction of the temperature dependence of the spectral slope in other samples. An example is presented in Fig. 13 where we plot $S_{V} / V^{2}$ vs $T$ at 1,10 , and $100 \mathrm{~s}^{-1}$. In this data set, we observe the usual broad peak, shifting to higher temperatures with measuring frequency, and thermally activated kinetics. However, from Fig. 14 we see that the DDH relation [Eq. (10)] clearly overestimates the variation of the spectral slope with temperature.

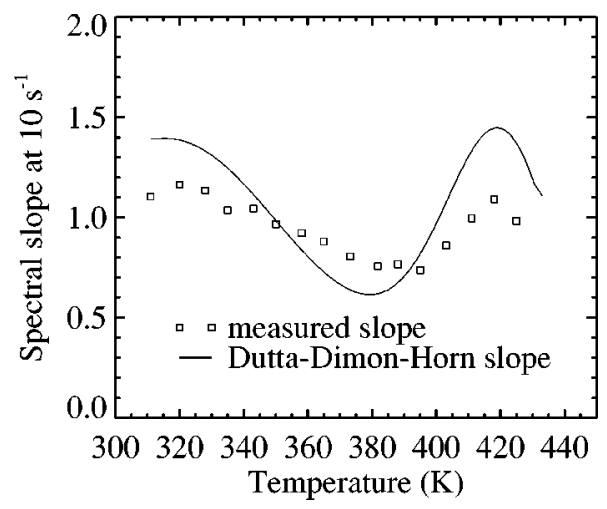

FIG. 14. Dutta-Dimon-Horn slope compared to the measured spectral slope in another sample. All features are clearly reproduced except that the variation of the measured slope with temperature is less pronounced. Note that the curves intersect at $350 \mathrm{~K}$ where the spectral slope equals 1 . 
What might be the cause of this discrepancy? Of course, one of the conditions of the DDH relation may not be met. We could suspect the variance to have an additional temperature dependence to the noise intensity. The model (Fig. 9), in fact, produces this, if the offset between the minima of the double potential well is much larger than $2 k_{B} T$ : the variance, $1 / \cosh ^{2}\left[\Delta E /\left(2 k_{B} T\right)\right]$, will then be temperature dependent. However, such a temperature dependence of $S(f, T)$ in the right-hand side of Eq. (10) will only offset the spectral slope to one direction, so that agreement can never be obtained. A rather complicated temperature dependence would have to be added for which the derivative changes sign exactly at the temperature where the spectral slope equals -1 . The physics that underlie this deviation from the DDH behavior is unclear. We stress, however, that the samples comply with the $\mathrm{DDH}$ criterion in every other respect.

\section{DISCUSSION}

So far, we have established that the observed resistance fluctuations are entirely produced by thermally activated processes and can be self-consistently described by a single distribution of activation energies. Until this stage, it appeared unnecessary to specify the underlying physical mechanism for the resistance noise observed. Prime candidates for producing resistance noise in $a-\mathrm{Si}: \mathrm{H}$ are fluctuations in the number of free carriers and mobility fluctuations due to, for example, structural (metastable) changes. The remarkable fact that the DDH analysis works out so well may provide an important clue to determine which noise source prevails. In the following sections we will discuss mobility fluctuations and number fluctuations as they may occur in $a-\mathrm{Si}: \mathrm{H}$.

\section{A. Mobility fluctuations and structural changes}

As a mobility fluctuation we take any fluctuation that produces a change in the resistance except, of course, fluctuations in the number of free carriers. Ionic motion, or defect creation and annihilation are examples of structural changes leading to mobility fluctuations that comply with the DDH conditions: The kinetics of these fluctuations are generally thermally activated with energy barriers that lie typically in the $\mathrm{eV}$ range. In all cases, disorder of the silicon network is expected to result in a sufficiently broad distribution of energies that yields characteristic rates accessible with our experimental setup. Further, the energy for defect creation and annealing are known to be very similar so that the well offset $\Delta E$ between the two configurational minima (Fig. 9) can be quite small ${ }^{25}$ and the criterion $\Delta E \lesssim 2 k_{B} T$ is probably met. The extensive noise study performed by Parman and Kakalios $^{4,6,7}$ on phosphorus-doped $a$-Si with coplanar contact geometry indicates that structural fluctuations dominate the noise in such devices. Inhomogeneous current paths, whose conductivity is sensitive to local rearrangements of the amorphous network, have been invoked to explain the anomalously large discrete resistance switching observed.

Another effective noise source in $a-\mathrm{Si}: \mathrm{H}$ is hydrogen motion. ${ }^{8}$ Today, it is widely recognized that the incorporation of hydrogen favorably modifies the bonding structure of $a$-Si by inserting itself into weak $\mathrm{Si}-\mathrm{Si}$ bonds, releaving strain and sharpening the distribution of band tail states.
Therefore, a moving hydrogen atom most certainly induces resistance noise through fluctuations in the electron or hole scattering rates. It may leave behind defects that even trap the charge carriers. In metals, noise spectroscopy has been shown to be very successful to study hydrogen hopping and diffusion. $^{26-31}$ Scofield and $\mathrm{Webb}^{26}$ measured resistance fluctuations in $\mathrm{Nb}$ films due to long-range hydrogen diffusion. Zimmerman and $\mathrm{Webb}^{28}$ observed hydrogen hopping between nearby lattice sites in $\mathrm{Pd}$ at low temperatures. The first noise data on hydrogen hopping in amorphous metals came from Alers et al. ${ }^{29}$ and Zimmerman et al. ${ }^{30}$ who studied hydrogen motion in amorphous $\mathrm{Ni}-\mathrm{Zi}$ and amorphous $\mathrm{Si}-\mathrm{Pd}$, respectively.

Long-range interactions as defect relaxation ${ }^{32-34}$ or collective hydrogen motion are two other suggested mechanisms operative to create mobility fluctuations in $a$-Si:H. In recent years, defect relaxation has been proposed to understand the results of transient photocurrent measurements. ${ }^{35}$ Defect relaxation may occur following a change in charge state of the defect, when the structure adapts itself to the new situation, accompanied by a shifting of the energy level and by resistance fluctuations.

Finally, changes in the occupancy of defects can be expected to produce mobility fluctuations: It is believed that the majority of defects is charged instead of neutral, leading to an electric field that changes over microscopic distances. Therefore, redistribution of the trapped charges leads to a fluctuating potential landscape in which the other carriers move, and hence to resistance fluctuations.

All these mechanisms have in common that in order to exactly calculate the noise one needs to know the coupling of the fluctuating entity to the measured resistance. Generally, this coupling is unknown, making it difficult, if not impossible, to give a clear assignment of the prevailing mechanism based on quantitative arguments. Fortunately, number fluctuations do not suffer so much from such free parameters since the variance can be related to the number of fluctuators that generally can be estimated with sufficient precision.

\section{B. Number fluctuations}

The number of free electrons and holes in the conduction and valence band of a semiconductor may fluctuate because of generation (emission) and recombination (trapping) processes connecting the bands and the traps. Fluctuations in the number of free carriers naturally cause fluctuations in the resistance. This type of noise is well known and, in the literature, usually referred to as generation-recombination noise. The term recombination refers to the annihilation of electrons and holes in one collision, but in this context also to simple carrier trapping without the actual recombination taking place. Hydrogenated amorphous silicon possesses a continuous distribution of traps spread throughout the mobility gap. In this section we will examine whether noise in $a-\mathrm{Si}: \mathrm{H}$ can be due to generation and recombination processes. We start by discussing a system involving two levels, which represents the simplest form of GR noise.

(i) GR noise involving two levels. We consider in a semiconductor an arbitrary number of traps at a single energy level, which interact with the conduction band only. This trap level is located at an energy $E_{t}$ from the valence band. 
The free-electron mobility edge is at $E_{c}$. We shall now examine the temperature dependence of GR noise and see whether it obeys the DDH criteria and possibly can account for our data.

The decay of an excess carrier concentration $\Delta N=N$ $-N_{0}$ to its average value $N_{0}$ is given by the following differential equation (see, for example, van der $\mathrm{Ziel}^{3}$ )

$$
\begin{aligned}
\frac{d}{d t} \Delta N & =g(N)-r(N)+H(t) \\
& =-\left[r^{\prime}\left(N_{0}\right)-g^{\prime}\left(N_{0}\right)\right] \Delta N+H(t),
\end{aligned}
$$

where $g(N)$ and $r(N)$ are the generation and recombination rate, respectively, and $H(t)$ is a Langevin noise term driving the fluctuations. The primes indicate derivatives with respect to $N$. The power spectrum for the relative fluctuations in $N$ is then given by

$$
\frac{S_{N}}{N^{2}}=4 \frac{\overline{\Delta N}^{2}}{N^{2}} \frac{\tau}{1+4 \pi^{2} f^{2} \tau^{2}},
$$

where

$$
\begin{aligned}
& \frac{1}{\tau}=r^{\prime}\left(N_{0}\right)-g^{\prime}\left(N_{0}\right), \text { and } \\
& \overline{\Delta N}^{2}=\frac{g\left(N_{0}\right)}{r^{\prime}\left(N_{0}\right)-g^{\prime}\left(N_{0}\right)} .
\end{aligned}
$$

Following van Rheenen, Bosman, and Zijlstra ${ }^{36}$ the generation and recombination rates read

$$
\begin{gathered}
g=\frac{1}{\tau_{e}} n_{T}, \\
r=\rho N\left(N_{T}-n_{T}\right) .
\end{gathered}
$$

Here, $N_{T}, n_{T}$, and $\rho$ represent the total number of traps, the number of occupied traps, and the trapping rate, respectively. The trapping rate is defined as the capture cross section of the trap $(\sigma)$ times the thermal velocity $\left(v_{\mathrm{th}}\right)$ per unit volume. The emission rate $1 / \tau_{e}$ is thermally activated with activation energy $E_{a}=E_{c}-E_{t}$ and attempt rate $1 / \tau_{0}$,

$$
\frac{1}{\tau_{e}}=\frac{1}{\tau_{0}} e^{-E_{a} /\left(k_{B} T\right)} .
$$

The charge-neutrality condition prescribes that the total number of carriers (trapped plus free carriers) is constant. Further, in equilibrium detailed balance requires that $g=r$ so that

$$
\frac{1}{\tau_{e}} n_{T 0}=\rho N_{0}\left(N_{T}-n_{T 0}\right),
$$

where the subscript 0 denotes thermal equilibrium values. Using the charge neutrality condition, Eq. (14), (16), and (18) can be combined to give

$$
\frac{1}{\tau}=\frac{1}{\tau_{e}}+\rho\left(N+N_{T}-n_{T}\right)=\frac{1}{\tau_{e}}+\frac{1}{\tau_{c}}+\frac{1}{\tau_{T}},
$$

where

$$
\frac{1}{\tau_{T}}=\rho\left(N_{T}-n_{T}\right)
$$

It is noted that when $\tau_{e}, \tau_{c}$, and $\tau_{T}$ differ very much, $\tau$ of course corresponds to the smallest of the three. In standard analyses, this third term is generally omitted but should be taken into account and is in fact rate limiting when the number of empty traps is much larger than the number of free electrons, as is the case in $a-\mathrm{Si}: \mathrm{H}$.

The variance is readily calculated from Eqs. (15), (16), and (18) and reads

$$
\left(\frac{1}{\overline{\Delta N}^{2}}\right)=\frac{1}{N}+\frac{1}{\left(N_{T}-n_{T}\right)}+\frac{1}{n_{T}} .
$$

For typical values of $1 / \tau_{0} \simeq 7 \times 10^{12} \mathrm{~s}^{-1}, E_{a} \simeq 0.7 \mathrm{eV}, T$ $=300 \mathrm{~K}, \sigma \simeq 10^{-15} \mathrm{~cm}^{2}, v_{\mathrm{th}} \simeq 10^{7} \mathrm{~cm} \mathrm{~s}^{-1}$, and taking for the effective density of states at the mobility edge $N_{c}$ $=10^{21} \mathrm{~cm}^{-3}$ so that $N / V \simeq 10^{9} \mathrm{~cm}^{-3}$ we obtain $1 / \tau_{e}$ $\simeq 10 \mathrm{~s}^{-1}$, and $1 / \tau_{c} \simeq 10 \mathrm{~s}^{-1}$. These rates are in the accessible range of frequencies. $1 / \tau_{T}$, however, is much larger, as can be calculated by integrating the electronic density of states distribution in amorphous silicon $\mathcal{N}(E)$ from the Fermi energy to the bottom of the conduction band:

$$
\frac{1}{\tau_{T}}=\rho \int_{E_{F}}^{E_{c}} \mathcal{N}(E) d E
$$

We find $1 / \tau \simeq 1 / \tau_{T} \sim 10^{12} \mathrm{~s}^{-1}$. This frequency, which is the typical frequency that shows up in the power spectra, lies far beyond the spectral domain of our experimental setup. In addition, $1 / \tau$ is not thermally activated and, at most, weakly temperature dependent. Two important conditions under which the DDH relation, which describes our data so well, is derived are not met. The number of free carriers is thermally activated so that the relative noise intensity due to trapping and emission of electrons strongly decreases with temperature, which we do not see. Secondly, the characteristic frequency $1 / \tau$ is not thermally activated, again in contrast to our findings. Further, assuming $T=300 \mathrm{~K}$, the relative noise intensity amounts to

$$
\frac{S_{V}}{V^{2}} \sim \frac{\tau}{N} \sim \frac{10^{-12}}{10^{3}}=10^{-15} \mathrm{~s},
$$

a value much smaller than the measured noise levels. Consequently we have to reject simple GR processes to explain our measurements.

We note that from this discussion it follows that a model proposed by Bathaei and Anderson for GR noise in $a-\mathrm{Si}: \mathrm{H}$ does not apply. Correctly they conclude that the capture rate is too fast to be observed but erroneously they continue by postulating that the observed low-frequency noise must be due to the emission processes that are thermally activated. We have just shown for $a$-Si:H that the effective rate is the sum of the capture and emission rates.

(ii) GR noise involving three levels. A more realistic model for $a-\mathrm{Si}: \mathrm{H}$ is constructed if we add a third level, namely, the valence band. This enables us to include recombination of electrons and holes at defects. Whereas in a twolevel system the spectrum is described by a Lorentzian char- 
acterized by a single time constant, carrier fluctuations between three levels involves two time constants: one to represent the extremely fast carrier trapping and emission processes as we have already encountered above, the other to characterize the recombination of electrons and holes, which is in general a much slower process. The recombination rate is inherently limited by the generation rate of the minority carriers that will generally be thermally activated, in agreement with our experimental findings. The variance in the number of free electrons can be straightforwardly calculated and is given by ${ }^{37,38}$

$$
\overline{\Delta N}^{2}=A\left(\frac{1}{P}+\frac{1}{n_{T}}+\frac{1}{N_{T}-n_{T}}\right),
$$

where $P$ is the number of free holes, and where

$$
A=\left[\frac{1}{N P}+\left(\frac{1}{n_{T}}+\frac{1}{N_{T}-n_{T}}\right)\left(\frac{1}{N}+\frac{1}{P}\right)\right]^{-1} .
$$

In the limit that $N_{T}-n_{T}$ and $n_{T}$ are much larger than $N$ and $P$ we find that $\overline{\Delta N}^{2} / N^{2} \simeq 1 / N$, i.e., equal to the inverse number of free electrons in the active part of the sample. We thus see that the inclusion of electron-hole recombination does produce a low-frequency term to the total power spectrum with a thermally activated rate constant, a quite pleasant circumstance since we observe this in our measurements. The variance, unfortunately, is strongly temperature dependent, disqualifying the three-level model as a satisfactory explanation for our data.

A next reasonable step in sophistication, quite appropriate for amorphous semiconductors, is to consider a system with recombination centers distributed throughout the gap.

(iii) GR noise involving a uniform distribution of trap levels. In general, for $m$ levels there will be $m-1$ time constants. ${ }^{39}$ Therefore, one might expect that in an amorphous semiconductor, with recombination centra distributed throughout the gap, a $1 / f$ spectrum will be obtained. Surprisingly, numerical calculations by Lee, Amberiadis, and Van der Ziel show that the power spectrum closely resembles that of a three-level system. The reason is that not all traps contribute equally to the variance, since traps more than $\sim 2 k_{B} T$ above $E_{F}$ are always empty, whereas those lying more than $\sim 2 k_{B} T$ below $E_{F}$ are always filled. Therefore, the contribution of individual trap levels to the noise should be weighted by a Fermi factor $F(1-F), F$ being the trap occupation probability. ${ }^{14}$ The authors consistently found a sum of two near-Lorentzian spectra with one time constant associated with the very fast electron capture $\left(\tau_{n}\right)$ and the other with the much slower recombination processes $\left(\tau_{R}\right)$. As for a three-level system, the former Lorentzian has a lowfrequency plateau at a much smaller intensity than the latter. This is schematically illustrated in Fig. 15. When the number of free electrons is smaller than $N_{T}$, the number of traps within $2 k_{B} T$ of the Fermi level, the total variance of the relative fluctuations associated with recombination processes turns out to be equal to $1 / N_{T}$. This fact was not explicitly noted in Lee's paper but it can readily be deduced from the spectra they present. In summary, a single recombination level produces a variance of the relative fluctuations equal to one over the number of free electrons. In contrast, uniformly distributed traps in thermal equilibrium lead to a spectrum

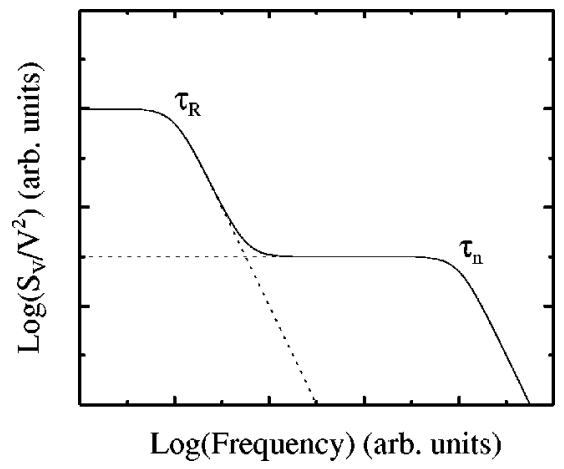

FIG. 15. Schematic representation of the power spectrum for fluctuations in the number of free electrons due to trapping and recombination at uniformly distributed traps.

similar to that of a three-level system but with markedly different magnitude and temperature dependence of the noise intensity. The observation implies that, here, the variance $1 / N_{T}$ is only weakly temperature dependent. This notion makes the GR model with a uniform distribution of recombination levels a promising candidate to explain our data, as we will see.

\section{Noise intensity}

The variance can be computed from the power spectra by integrating over frequency. The integration can be performed with sufficiently high accuracy due to the strong deviation from a pure $1 / f$ spectrum. Quite fortunately, we find for the variance a very reasonable value:

$$
\operatorname{var}=\int \frac{S_{V}}{V^{2}} d f=3 \times 10^{-10}
$$

Indeed, for a sample volume of $7 \times 10^{-6} \mathrm{~cm}^{3}$, namely, we obtain an empirical value for the density of states of

$$
D\left(E_{F}\right)=\frac{1}{\left(\operatorname{var} V 4 k_{B} T\right)} \sim 5 \times 10^{15} \mathrm{~cm}^{-3}(\mathrm{eV})^{-1},
$$

which is in reasonable agreement with the density of states generally found for high-quality thick intrinsic $a$-Si:H layers. We would, however, expect to find a defect density about one order of magnitude larger in our devices in which the intrinsic film is sandwiched between two highly doped buffer layers of $a$-Si:H. We note again that the number traps around the Fermi level, and thus the variance, depends only weakly (linearly) on temperature, in agreement with our observations.

We have some difficulty understanding why $S_{V} / V^{2}$ increases upon light soaking (see Fig. 8). From the foregoing discussion we would expect $S_{V} / V^{2}$ to decrease following degradation by illumination, since the density of traps increases, predominantly in the midgap region, where the Fermi level is located. This observation, together with the finding that the defect density suggested by the theory is a bit low, possibly suggests that recombination produces additional resistance fluctuations other than number fluctuations. Next to number fluctuations, recombination also involves fluctuations in the charge state of traps and therefore produces fluctuations in the potential landscape in which the 


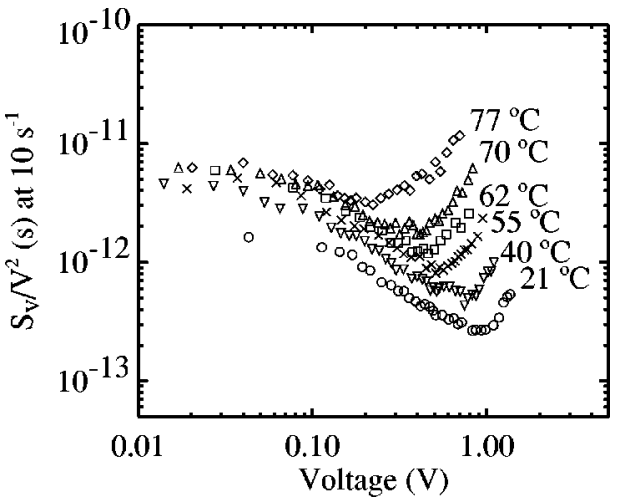

FIG. 16. Relative noise power at $10 \mathrm{~s}^{-1}$ vs voltage with temperature as parameter. The plateau region and the suppression effect are present in all samples. The onset of the anomalous increase at high voltage or high temperatures is sample dependent.

free carriers move. In other words, recombination leads to mobility fluctuations due to scattering of free carriers in a fluctuating potential landscape having the same kinetics as number fluctuations. The larger the number of recombination centers, the larger the intensity of the mobility fluctuations induced by these centers.

Finally, we stress that the numerical calculations by Lee et al. rely on a strictly uniform distribution of trap levels. In $a-\mathrm{Si}: \mathrm{H}$ the situation is of course different since there are additional tails of electronic levels in the gap exponentially increasing towards the conduction and the valence band. Although we do not expect the incorporation of a more realistic density of states to result in a rise of the noise intensity after degradation, it would be most interesting to examine the effect of the density of states distribution on the variance.

\section{Distribution of activation energies}

The recombination rate is evidently limited by the generation rate of the minority carriers, i.e., the holes. We can now identify the measured activation energy of $f_{1}$ with an energy barrier limiting the hole emission. Since exclusively the traps within $\sim 2 k_{B} T$ of the Fermi energy are effective fluctuators we expect that only a band of emission energies of width $\sim 4 k_{B} T$ will be observed. At room temperature $k_{B} T$ is only $25 \mathrm{meV}$. From the DDH analysis, we obtain $0.2 \mathrm{eV}$, which is twice $4 k_{B} T$. This relatively broad distribution of activation energies can simply be accounted for by the considerable band bending in our devices. Simulations of the energy-band diagram for sandwich devices (recall Fig. 1) allow for a width of $0.2 \mathrm{eV}$. Taking into account this sample inhomogeneity, also the shape of the spectrum is consistent with the model for GR noise in an amorphous semiconductor.

\section{CONCLUSION}

We showed that the relative resistance fluctuations in $a-\mathrm{Si}: \mathrm{H}$ are independent of current from 290 to $420 \mathrm{~K}$ as expected in a device with an Ohmic current-voltage characteristic. A study of the noise statistics did not reveal any indications of non-Gaussianity, in contrast to results reported in the literature on doped $n$-type $a$-Si:H taken, however, under markedly different experimental conditions. It was demonstrated phenomenologically how the temperature and

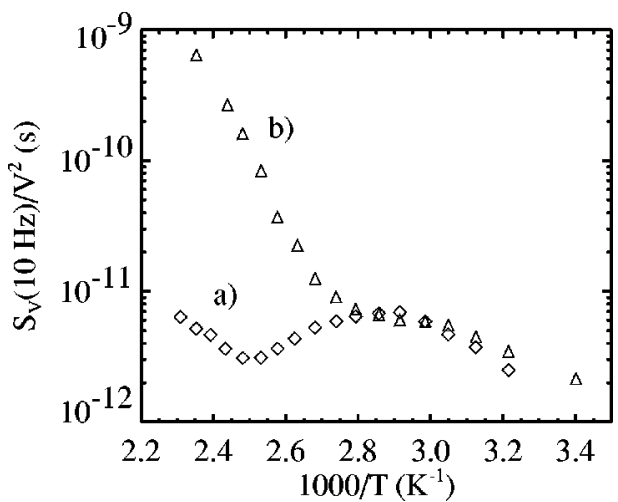

FIG. 17. Relative noise power at $10 \mathrm{~s}^{-1}$ vs temperature. Curve $a$ was taken from Fig. 13. Curve $b$ is taken in a different sample and shows the anomalous rise of $S_{V} / V^{2}$.

spectral dependences of the relative noise intensity can be condensed into one single graph of the distribution of activation energies. This was achieved in the DDH approach that decomposes spectra into Lorentzians with thermally activated rate constants. The resulting distribution peaks at 0.85 $\mathrm{eV}$ with a FWHM of $\sim 0.2 \mathrm{eV}$. An attempt rate of 7 $\times 10^{12} \mathrm{~s}^{-1}$ is found, a realistic phonon frequency in this material.

As to the underlying physics, the tentative conclusion is that a GR noise model for distributed trap levels is consistent with our experimental observations: The variance is virtually temperature independent, while the characteristic rate constants are thermally activated so that a DDH type of analysis that covered our data so nicely is appropriate for this model. The distribution of activation energies is wider than $\sim 4 k_{B} T$ but is readily accounted for by the position dependence of the energy-band diagram as it exists in thin-film devices with sandwich geometry. Even the absolute magnitude of the relative noise intensity finds a quantitative explanation in terms of the number of traps around the Fermi level. We, however, realize that there is a problem associated with the effect of degradation on the relative noise intensity. Possibly, fluctuations in the trapped charge density produce resistance noise with the same kinetics as but with intensities that can not be explained by standard number fluctuations. Although experimental evidence points to GR noise, we are still searching for decisive arguments. In our further quest to uncover the origin for noise in $a-\mathrm{Si}: \mathrm{H}$, we decided to study resistance fluctuations under nonthermal equilibrium conditions, i.e., under steady-state illumination and carrier injection. This allows us to tune the (quasi) Fermi energy of electrons and holes and perform a new critical test. We report on these experiments in the accompanying paper.

\section{ACKNOWLEDGMENTS}

We thank F.J.M. Wollenberg, R. Beelen, and P. Jurrius for their invaluable technical assistance. We acknowledge C.H.M. van der Werf and R.E.I. Schropp for supplying the amorphous silicon films. We would also like to thank W.F. van der Weg for stimulating discussions.

\section{APPENDIX: ANOMALOUS EFFECTS}

Despite the most careful sample preparation methods to avoid "dirty" contacts, we wished to meticulously examine 


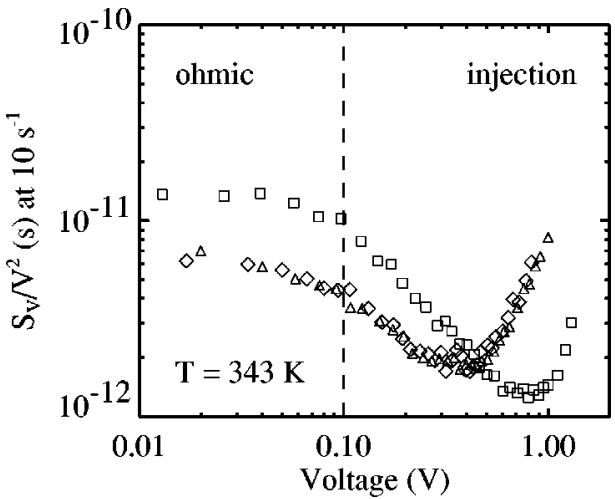

FIG. 18. $S_{V} / V^{2}$ vs voltage for the annealed sample $(\triangle)$, the sample after degradation $(\square)$, and after annealing to the original state $(\diamond)$.

whether the data did or did not suffer from noise contributions from sources other than those in the intrinsic $a-\mathrm{Si}: \mathrm{H}$ layer. In Fig. 16 the relative noise intensity is plotted versus voltage for six values of the temperature. This plot gives a representative example of the anomalous behavior of excess noise observed in some of our devices. In the Ohmic regime, i.e., for voltages $<0.1 \mathrm{~V}, S_{V} / V^{2}$ is constant. This is expected for resistance noise and has been discussed in Sec. III. Further, under low injection conditions we observe space charge suppression of the noise that complies with previous results as well (see Fig. 4). However, above certain applied electric fields, depending on temperature, $S_{V} / V^{2}$ steeply increases with voltage. This effect is practically always observed, though the turning point varies from sample to sample. We discovered that the noise becomes unstationary, particularly in samples where the onset of the steep increase occurs at the highest voltages. In such cases we frequently observe random telegraph signals with a highly unstable switching rate. From Fig. 16 it is seen that at $77^{\circ} \mathrm{C}$ the anomalous rise sets in already close to the Ohmic regime. In order to see how $S_{V} / V^{2}$ evolves as a function of temperature in the Ohmic regime, we measured $S_{V} / V^{2}$ at $10 \mathrm{~s}^{-1}$ vs $T$ at a constant voltage of $50 \mathrm{mV}$. The result is shown in Fig. 17, curve $b$. At low temperatures, the relative noise intensity virtually tracks the usual curve found in all samples (curve $a$ ). However, in this case around $90{ }^{\circ} \mathrm{C}$ the relative noise intensity in the Ohmic regime sharply rises, in contrast to what has been observed in the other samples. The rise sets in at exactly the temperature where the turning point of $S_{V} / V^{2}$ vs $V$ enters the Ohmic regime (see Fig. 16).

We have also examined the effect of degradation as a result of white-light illumination. In Fig. 18 we show three data sets. The triangles represent $S_{V} / V^{2}$ for the device in the annealed state. After degradation, the relative noise power increases and we see that the turning point has shifted to higher voltages. We checked that subsequent annealing reduces the noise to the original values.

We now prove that the conspicuous rise of $S_{V} / V^{2}$ is not related to GR noise. It appears that the anomalous contribution to the noise simply tracks the sample conductivity. In Fig. 19 all the anomalous excess noise observed in the measurements of $S_{V} / V^{2}$ vs $T$ and $V$, both in the annealed state and after degradation is collected and plotted versus the con-

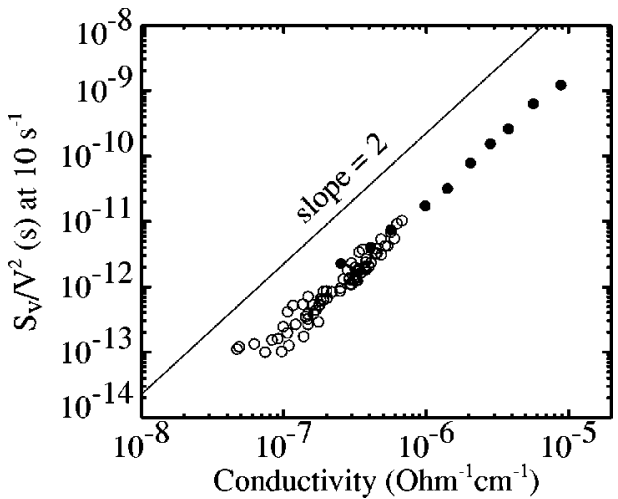

FIG. 19. Anomalous noise intensity, i.e., the actually measured noise intensity minus the GR noise component, vs sample conductivity. Strong correlation between $\sigma$ and $S_{V} / V^{2}$ over nearly five orders of magnitude points to contact noise.

ductivity. The filled symbols are from Fig. 17, and the open symbols from measurements of $S_{V} / V^{2}$ versus $V$. As can be seen in the figure, one curve tracks all these measurements, showing that $S_{V} / V^{2}$ is proportional to the conductivity squared over nearly five orders of magnitude.

What can be the origin of this remarkable correlation between the anomalous contribution to $S_{V} / V^{2}$ and the conductivity? A straightforward interpretation in terms of contact noise can be found. Let us represent the sample by a contact resistance $R_{c}$ and a much larger bulk resistance $R_{b}$ in series. A noise source in the contact region is assumed to produce a resistance fluctuation $\delta R_{c}$, which we take to be independent of temperature. Then, the relative voltage fluctuations will be

$$
\frac{(\delta V)^{2}}{V^{2}}=\frac{\left(\delta R_{c}\right)^{2}}{\left(R_{c}+R_{b}\right)^{2}} \simeq \frac{\left(\delta R_{c}\right)^{2}}{\left(R_{b}\right)^{2}} .
$$

This would produce the required quadratic dependence on the conductivity of the anomalous contribution to $S_{V} / V^{2}$.

We note, however, that the nonstationary effects that we observe resemble the non-Gaussian noise observed in doped $n$-type $a$-Si:H layers, ${ }^{6} a$-Si:H Schottky barriers, ${ }^{10}$ or heavily doped $p$-type $\mathrm{Cr}-p^{+}-\mathrm{Cr}$ sandwich structures. ${ }^{40}$ The observations might very well have a physical explanation in that at high temperatures, where the conductivity of the intrinsic layer approaches the conductivity of the doped buffer layers, the noise from these buffer layers dominates. We have checked that the noise is not in the leads or the electrically conductive adhesive on the chromium contact pads. The anomalous noise contribution may arise from sources that reside in the metal film, the doped contact layers, or in the intrinsic layer. Since a very trivial explanation in terms of contact noise can be given we will treat it as such. We conclude that a device with a thicker intrinsic layer or with coplanar geometry should be chosen if one wishes to study noise in intrinsic $a-\mathrm{Si}: \mathrm{H}$ at high temperatures. 
${ }^{1}$ D. Staebler and C. Wronski, Appl. Phys. Lett. 31, 292 (1977).

${ }^{2}$ R. Street, Hydrogenated Amorphous Silicon (Cambridge University Press, Cambridge, 1991).

${ }^{3}$ A. van der Ziel, Noise in Solid State Devices and Circuits (John Wiley and Sons, New York, 1986).

${ }^{4}$ C.E. Parman and J. Kakalios, Phys. Rev. Lett. 67, 2529 (1991).

${ }^{5}$ C.E. Parman, N.E. Israeloff, and J. Kakalios, Phys. Rev. B 44, 8391 (1991).

${ }^{6}$ C.E. Parman, N.E. Israeloff, and J. Kakalios, Phys. Rev. Lett. 69, 1097 (1992).

${ }^{7}$ C.E Parman, N.E. Israeloff, and J. Kakalios, Phys. Rev. B 47, 12578 (1993).

${ }^{8}$ J. Fan and J. Kakalios, Philos. Mag. B 69, 595 (1994).

${ }^{9}$ C. Rogers, R. Buhrman, H. Kroger, and L. Smith, Appl. Phys. Lett. 49, 1107 (1986).

${ }^{10}$ R. Arce, L. Ley, and M. Hundhausen, J. Non-Cryst. Solids 114, 696 (1989).

${ }^{11}$ N. Bernhard, B. Frank, and G. Bauer, J. Non-Cryst. Solids 164166, 473 (1993).

${ }^{12}$ T. Teuschler, M. Hundhausen, L. Ley, and R. Arce, Phys. Rev. B 47, 12687 (1993).

${ }^{13}$ T. Teuschler, M. Hundhausen, and L. Ley, J. Appl. Phys. 75, 2690 (1994).

${ }^{14}$ M. Baciocchi, A. D'Amico, and C. van Vliet, Solid-State Electron. 34, 1439 (1991).

${ }^{15}$ Z. Bathaei and J. Anderson, Philos. Mag. B 55, 87 (1987).

${ }^{16}$ M. Nelkin and A.-M. Tremblay, J. Stat. Phys. 25, 253 (1981).

${ }^{17}$ P. Restle, M. Weissman, and R. Black, J. Appl. Phys. 54, 5844 (1983)

${ }^{18}$ G. Khera, J. Kakalios, Q. Wang, and E. Iwaniczko, in Amorphous Silicon Technology, edited by M. Hack et al., MRS Symposia Proceedings No. 420 (Materials Research Society, Pittsburgh, 1996), p. 641.
${ }^{19}$ R. Johanson, D. Scansen, and S. Kasap, Philos. Mag. B 73, 707 (1996).

${ }^{20}$ M. Stutzmann, W.B. Jackson, and C. Tsai, Phys. Rev. B 32, 23 (1985).

${ }^{21}$ A. van der Ziel, Physica A 16, 359 (1950).

${ }^{22}$ P. Dutta, P. Dimon, and P.M. Horn, Phys. Rev. Lett. 43, 646 (1979).

${ }^{23}$ M.B. Weissman, Rev. Mod. Phys. 60, 537 (1988).

${ }^{24}$ P. Dutta and P. Horn, Rev. Mod. Phys. 53, 497 (1981).

${ }^{25}$ R.S. Crandall, Phys. Rev. B 36, 2645 (1987).

${ }^{26}$ J.H. Scofield and W. Webb, Phys. Rev. Lett. 54, 353 (1985).

${ }^{27}$ J.H. Scofield, J.V. Mantese, and W. Webb, Phys. Rev. B 34, 723 (1986).

${ }^{28}$ N.M. Zimmerman and W.W. Webb, Phys. Rev. Lett. 61, 889 (1988).

${ }^{29}$ G.B. Alers, M.B. Weissman, R.S. Averback, and H. Shyu, Phys. Rev. B 40, 900 (1989).

${ }^{30}$ N.M. Zimmerman and W.W. Webb, Phys. Rev. Lett. 65, 1040 (1990).

${ }^{31}$ B.D. Nevins and M.B. Weissman, Phys. Rev. B 41, 1301 (1990).

${ }^{32}$ J.D. Cohen, T.M. Leen, and R.J. Rasmussen, Phys. Rev. Lett. 69, 3358 (1992).

${ }^{33}$ F. Zhong and J. Cohen, Phys. Rev. Lett. 71, 597 (1993).

${ }^{34}$ H. Branz and E. Schiff, Phys. Rev. B 48, 8667 (1993).

${ }^{35}$ D. Han, D.C. Melcher, E.A. Schiff, and M. Silver, Phys. Rev. B 48, 8658 (1993).

${ }^{36}$ A. van Rheenen, G. Bosman, and R. Zijlstra, Solid-State Electron. 30, 259 (1987).

${ }^{37}$ R. Burgess, Proc. Phys. Soc. London, Sect. B 86, 661 (1955).

${ }^{38}$ K. van Vliet, Physica (Amsterdam) 23, 248 (1957).

${ }^{39}$ K. van Vliet and J. Fassett, Fluctuation Phenomena in Solids (Academic Press, New York, 1965), pp. 267-354.

${ }^{40}$ W. Choi, A. Owen, P. Lecomber, and M. Rose, J. Appl. Phys. 68, 120 (1990). 